\title{
The importance of resilience in adolescent mental health promotion and risk behaviour prevention
}

\author{
Krzysztof Ostaszewski ${ }^{1}$
}

Received: 1 October 2020 / Accepted: 2 October 2020 / Published online: 13 October 2020

(C) Swiss School of Public Health (SSPH+) 2020

From a health point of view, adolescence is one of the best periods in life. It is a time of peak health and strength. But, on the other hand, during this period of life there is the greatest tendency to undertake risky behaviours that compromise adolescents health and well-being. This is also the time, when young people become more vulnerable for mental problems including depression, suicidal behaviours, eating disorders, substance use and other addictive behaviours. The successful or unsuccessful development of adolescents depends on the risks and protection they are exposed to. The interplay between risk and protection influences the mechanisms of resilience or vulnerability. Therefore, support should be given to research on positive factors that contribute to adolescent resilience.

The theory of resilience provides a framework for understanding why some adolescents who are exposed to a number of risks do not develop negative health or social outcomes, and contrary to expectations, grow up successfully (Fergus and Zimmerman 2005). The concept of resilience includes compensatory and protective models to explain how positive factors operate to help adolescents overcome risks. Compensatory model indicates "positive factors" that offset the negative effect of risk factors. Whereas the protective factor model explains the "protective factors" that moderate the relationship between the risk factors and the negative outcomes. These two types of factors are known in the literature under two terms "protective factors" or "promotive factors" (Sameroff et al. 2003; Zimmerman et al. 2013).

This Editorial is part of the special issue Adolescent health in Central and Eastern Europe.

Krzysztof Ostaszewski ostasz@ipin.edu.pl

1 Department of Public Health, Institute of Psychiatry and Neurology, Warsaw, Poland
The knowledge on "protective" or "promotive" factors radically changed the approaches used in adolescent mental health promotion and risk behaviours prevention. Strengthbased approaches concentrate on providing youth with the internal and external attributes needed for healthy development (Bernat and Resnick 2006). There are a few strength-based concepts that are especially valuable in the context of adolescent mental health and well-being. They include supportive school environment, parent-child effective communication, meaningful extracurricular activities. These three examples were selected because they cut across problems addressed in this volume of IJPH.

Adolescents have the potential to control their development and actively influence the adaptation processes because of their exceptional plasticity (Lerner et al. 2003). This is particularly important in those cases where the development of a young person is heading in a wrong direction. We know that youth development is not a linear process and highly context-dependent. However, the use of developmental potential depends on the availability of prodevelopmental resources in the young person's environment. Examples of resources that should be integrated in a strength-based policy to support positive youth development are briefly presented below.

Supporting teacher-student relationships is associated with good school achievements, pro-social students involvement, better mental health status and lower level of risk behaviours. This notion is supported by a growing body of evidence from different cultures and countries including countries from CEE. Caring and supportive relationships with adults in school provide youth with the social support and emotional security needed to achieve a variety of developmental outcomes. Quality of studentteacher relationships is also one of the key components of positive school climate and student's school bonding. These school-related promotive factors help students to feel connected to other students and to adapt more easily to social norms and expectations. 
Parent-child supportive relationship is the most influential promotive factors in the context of youth positive development (Masten and Barnes 2018). Effective parenting during adolescence includes supervision and monitoring, emotional support, communication and positive modelling. Proper communication with an adolescent child plays key role in effective parenting, because creates proper family conditions that allow parents to recognize child's stressful or traumatic experiences and help to cope with them. New media are increasingly becoming the source of the unwanted experiences that pose a risk to adolescent mental health. Programs designed for youth may benefit from targeting positive parenting practices, and specifically parental communication skills.

Constructive use of leisure time in adolescence such as involvement in extracurricular activities, after-school programs, pro-social activities in social media contribute to positive development (Feldman Farb and Matjasko 2012). These kinds of activities offer an outlet for adolescents to learn social roles and psychosocial competencies. Also, provide opportunities to practice leadership, teamwork and decision making. These activities help youth navigate through adolescent-specific developmental needs and tasks, because participation in organized activities may help youth to gain autonomy and explore identity.

A solid foundation is needed for promotion and prevention models to serve as guidelines for decision/policy makers, program deliverers and evaluators. The knowledge on adolescent resilience and promotive factors creates this foundation. Important promotive factors (like three above mentioned) when available in adolescent lives affect their personal and social development in a way that supports their current well-being and successful transition to adulthood, including family life, pro-social involvement and personal growth.

The collection of articles published in this special issue is a unique portrait of mental health, lifestyles, health and risky behaviours of young people from CEE. This is particularly valuable because adolescents studied in this special issue represent one of the first generations of youth raised under new political, economic and societal rules. It is possible that resilience differs somewhat in this region depending on the socio-political context, especially regarding changes in schools, health care system, local communities and families. Opportunities for well-informed mental health promotion and strength-based prevention may both increase in number and importance as the modernization of CEE countries continues to develop.

\section{Compliance with ethical standards}

Conflict of interest The author declares that he/she has no conflict of interest.

\section{References}

Bernat D, Resnick M (2006) Healthy youth development: science and strategies. J Public Health Manag Pract November(Suppl):S10 S16

Feldman Farb A, Matjasko J (2012) Recent advances in research on school-basedextracurricular activities and adolescent development. Dev Rev 32(1):1-48

Fergus S, Zimmerman M (2005) Adolescent resilience: a framework for understanding healthy development in the face of risk. Ann Rev Public Health 26:399-419

Lerner R, Dowling E, Anderson P (2003) Positive youth development: thriving as the basis of personalhood and civil society. Dev Sci 7(3):172-180

Masten AS, Barnes AJ (2018) Resilience in children: developmental perspectives. Children (Basel, Switzerland) 5(7):98. https://doi. org/10.3390/children5070098

Sameroff A, Gutman L, Peck S (2003) Adaptation among youth facing multiple risks. In: Luthar S (ed) Resilience and vulnerability. Adaptation in the context of childhood adversities. Cambridge University Press, Cambridge, pp 364-391

Zimmerman M, Stoddard S, Eisman A, Caldwell C, Aiyer S, Miller A (2013) Adolescent resilience: promotive factors that inform prevention. Child Dev Perspect 7(4):215-220

Publisher's Note Springer Nature remains neutral with regard to jurisdictional claims in published maps and institutional affiliations. 